\title{
Introducing Hierarchy in Energy-Efficient Power Control Games
}

\author{
Yezekael Hayel \\ Lab. Informatique d'Avignon \\ Universite d'Avignon \\ 84911, Avignon, France \\ yezekael.hayel@univ- \\ avignon.fr
}

\author{
Samson Lasaulce \\ Lab. des Signaux et Systemes \\ CNRS - Supelec - Paris Sud \\ 91190, Gif-sur-Yvette, France \\ lasaulce@lss.supelec.fr
}

\author{
Merouane Debbah \\ Alcatel-Lucent Chair \\ Supelec \\ 91190, Gif-sur-Yvette, France \\ merouane.debbah@supelec.fr
}

\author{
Rachid El-Azouzi \\ Lab. Informatique d'Avignon \\ Universite d'Avignon \\ 84911, Avignon, France \\ rachid.elazouzi@univ- \\ avignon.fr
}

\begin{abstract}
We consider a multiple access channel where the users choose their best power control strategy in order to selfishly maximize their energy-efficiency. To increase the utilities with respect to the classical non-cooperative game, we introduce hierarchy in two ways. On the one hand, assuming singleuser decoding at the receiver, we investigate a Stackelberg formulation of the game where one user is the leader. On the other hand, assuming neither leader nor followers among the users, we introduce hierarchy by using successive interference cancelation at the receiver. For both cases, we study the existence and uniqueness of an equilibrium and compare the individual performance obtained in the hierarchical game with that obtained in the non-cooperative game. An exhaustive comparative analysis of the two games is also conducted. In order to optimize the choice of the leader in the Stackelberg formulation (with single user decoding) and that of the decoding order (in the non-cooperative game with successive interference cancelation), we study two measures of global energy-efficiency for the network and discussions are provided for each case.
\end{abstract}

\section{General Terms}

Game theory, Wireless Communications.

\section{Keywords}

Energy-efficiency, multiple access channel, Nash equilibrium, power control game, Stackelberg equilibrium.

\footnotetext{
*This work was partially supported by the INRIA ARC Program POPEYE and the BIONETS European project.
}

Permission to make digital or hard copies of all or part of this work for personal or classroom use is granted without fee provided that copies are not made or distributed for profit or commercial advantage and that copies bear this notice and the full citation on the first page. To copy otherwise, to republish, to post on servers or to redistribute to lists, requires prior specific permission and/or a fee.

GameComm 2008 October 20, 2008, Athens, GREECE.

Copyright (C) 2008 ICST ISBN \# 978-963-9799-31-8.

\section{INTRODUCTION}

In this paper, we consider a decentralized multiple access channels (MAC). By definition [1], the MAC consists of a network of several transmitters and one receiver. The network is said to be decentralized in the sense that the receiver does not dictate to the users their transmit power levels. Hence, each user can choose freely its power control policy in order to selfishly maximize a certain individual performance criterion, called utility (or payoff) in the context of game theoretic studies. Unlike many works concerning this problem, the chosen users' utility is not the transmission rate (e.g. $[2,3,4])$ but the energy-efficiency of their communications. The latter approach, which consists in maximizing the ratio of the net number of information bits that are transmitted without error per time unit to the transmit power level, has been introduced in [5] for flat fading channels and recently re-used by [6] for multi-carrier CDMA (code division multiple access) systems and linear receivers, motivated by the facts that mobile terminals have a limited battery life and where in some applications (e.g. a sensor network measuring a temperature field) the main concern is not the transmission rate.

As mentioned in [5] the Nash equilibrium (NE) in such games can be very energy inefficient. This is why [7] proposed, for multiple access channels with flat fading links and single-user decoding (SUD), a pricing mechanism to obtain improvements in the users' utilities with respect to the case with no pricing. To our knowledge, since the release of [7], no alternative way of tackling this problem in the context of energy-efficient power control games has been proposed. In this paper we propose an alternative approach to [7] for improving the network efficiency by considering a Stackelberg formulation of the problem when single-user decoding is assumed at the receiver and also by using a more efficient (and non-linear) decoding scheme namely successive interference cancelation (SIC). As we will see, in both cases the receiver only broadcasts common messages and the corresponding amount of additional signalling is reasonable. Note that the Stackelberg formulation arises naturally in some context of practical interests. For example, this hierarchy is naturally present in contexts where there are primary (licensed) users 
and secondary (unlicensed) users who can sense their environment because there are equipped with a cognitive radio $[8,9]$. It is also natural if the users access to the medium in an asynchronous manner. Note that there have been many works on Stackelberg games, even in the context of cognitive radio [10], but they do not consider energy-efficiency for the individual utility as defined in $[5,6,11]$. They rather consider transmission rate-type utilities (see e.g. $[12,13,3]$ ).

This paper is structured as follows. The general signal model is provided in Sec. 2. Sec. 3 reviews the main results of [6] for the non-cooperative game. Then, in Sec. 4 we introduce our Stackelberg formulation by arbitrarily choosing a game leader, when SUD is assumed. In Sec. 5 we consider a different receiver namely a successive interference canceler, for which an arbitrary decoding order is chosen on each block of data (or packets). The choice of the best leader and decoding order in terms of overall network energy-efficiency is discussed in Sec. 6. In Sec. 7 we provide numerical results to illustrate the theoretical results derived in the previous sections. Additional comments and possible extensions of this work are provided in Sec. 8 .

\section{SIGNAL MODEL}

We consider a decentralized multiple access channel with $K$ users. Note that for sake of clarity and in order to have a deep understanding of the addressed issues, this paper is essentially dedicated to the game with two players. When the $K$ - user case $(K>2)$ is as tractable as the 2-user case, the results will be written in the most general case. Otherwise, this will always be mentioned explicitly. We assume that the users transmit their data over block Rayleigh flat fading channels and that the receiver knows on each block the channel gains (coherent communication assumption) whereas each transmitter has only access to the knowledge of its own channel. The latter assumption is realistic in systems where the uplink-downlink channel reciprocity is valid or if a reliable feedback mechanism is available. Clearly we only consider the framework of non-cooperative games with complete information that is each rational user knows the utilities of the others. The equivalent baseband signal received by the base station can be written as

$$
Y=\sum_{i=1}^{K} h_{i} X_{i}+Z
$$

with $\forall i \in\{1, \ldots, K\}, \mathbb{E}\left|X_{i}\right|^{2}=p_{i},\left|h_{i}\right|$ is a Rayleigh distributed random variable and $Z \sim \mathbb{C N}\left(0, \sigma^{2}\right)$. The channel gains $h_{i}$ vary over time but are assumed to be constant over each block.

\section{REVIEW OF THE NON-COOPERATIVE GAME}

In the system under investigation, users are selfish in the sense of their energy-efficiency. Here we review a few key results $[6,7]$ concerning the non-cooperative game, which we will use to analytically evaluate the benefits from introducing hierarchy in this game.

For any user $i \in\{1, \ldots, K\}$ the receive single-user signalto-noise plus interference ratio (SINR) expresses as

$$
S I N R_{i}=\frac{p_{i}\left|h_{i}\right|^{2}}{\sum_{j \neq i} p_{j}\left|h_{j}\right|^{2}+\sigma^{2}}
$$

where $j \neq i$. The strategy of user $i \in\{1, \ldots, K\}$ consists in choosing its transmit power level $p_{i}$ in order to maximize its utility function which is chosen to be:

$$
u_{i}\left(p_{1}, \ldots, p_{K}\right)=\frac{T_{i}}{p_{i}}=\frac{R_{i} f\left(S I N R_{i}\right)}{p_{i}}
$$

where $f$ is an efficiency function representing the packet success rate and $R_{i}$ is the transmission rate $[5,6]$. By definition of the utility (Eq. (3)) we see that the frequency at which the power control is updated is chosen to be the reciprocal of the data block duration. When it exists, the Nash equilibrium of this game is given by

$$
\forall i \in\{1, \ldots, K\}, p_{i}^{N E}=\frac{\sigma^{2}}{\left|h_{i}\right|^{2}} \frac{\beta^{*}}{1-(K-1) \beta^{*}}
$$

where $\beta^{*}$ is the positive solution of the equation

$$
x f^{\prime}(x)=f(x) .
$$

This type of equation has a positive solution if the function $f$ is sigmoidal [14], which is what is assumed in this paper. The existence of a (non-trivial) Nash equilibrium is insured provided that $\beta^{*}<\frac{1}{K-1}$. Note that if $\beta^{*} \geq \frac{1}{K-1}$, this game has still a Nash equilibrium if the transmit power is limited i.e. $p_{i} \in\left[0, P_{\max }\right]$. Therefore if the users have not enough power to reach the SINR $\beta^{*}$ they will all transmit at their maximum power, which is also an equilibrium. In this paper, for sake of clarity, we will only consider the most interesting (and non-trivial) regime where the transmit powers are less than their maximal levels but all the results provided in this paper easily extend to the case of finite powers. In this regime, even if a user has an infinite transmit power he will not necessarily use all of it. This is what Eq. (4) shows: each player tunes its transmit power in order for his SINR to be equal to $\beta^{*}$. In the sequel, we will denote by $u_{i}^{(N E)}$ the energy efficiency obtained by each player $i$ at the NE.

Note that the problem formulation presented in this paper can be applied to other types of systems, so our analysis is not exclusively applicable to the signal model defined by Eq. (1). For example, in flat fading CDMA systems, the SINR after despreading (denoted by $S I \tilde{N} R_{i}$ ) of the received signal can be written in the same form as Eq. (2):

$$
S I \tilde{N} R_{i}=\frac{\tilde{p}_{i}\left|h_{i}\right|^{2}}{\frac{\sum_{j \neq i} \tilde{p}_{j}\left|h_{j}\right|^{2}}{N}+\sigma^{2}} .
$$

where $N$ is the spreading factor (also the processing gain) of the CDMA system. The strategy of user $i$ consists in choosing its transmit power level $\tilde{p}_{i}$ in order to maximize its utility function which is chosen to be:

$$
u_{i}\left(\tilde{p}_{1}, \ldots, \tilde{p}_{K}\right)=\frac{T_{i}}{\tilde{p}_{i}}=\frac{\tilde{R}_{i} \tilde{f}\left(S I \tilde{N} R_{i}\right)}{\tilde{p}_{i}}
$$

where $\tilde{f}$ is an efficiency function representing the packet success rate. The study of the case of CDMA system can be directly obtained from the signal model used in this paper by observing that the two models are merely linked by the following change of variables: $p_{i}=\frac{\tilde{p}_{i}}{N}, R_{i}=\frac{\tilde{R}_{i}}{N}$ and $f(x)=\tilde{f}(N x)$, the $S I N R$ of user $i$ becomes :

$$
S I \tilde{N} R_{i}=\frac{N p_{i}\left|h_{i}\right|^{2}}{\sum_{j \neq i} p_{j}\left|h_{j}\right|^{2}+\sigma^{2}}
$$


and its utility can be rewritten as

$$
u_{i}\left(p_{1}, \ldots, p_{K}\right)=\frac{\tilde{R}_{i} \tilde{f}\left(N . S I N R_{i}\right)}{\tilde{p}_{i}}=\frac{R_{i} f\left(S I N R_{i}\right)}{p_{i}}
$$

where

$$
S I N R_{i}=\frac{p_{i}\left|h_{i}\right|^{2}}{\sum_{j \neq i} p_{j}\left|h_{j}\right|^{2}+\sigma^{2}} .
$$

This clearly establish the link between our signal model and that used by [6] for CDMA systems and flat fading channels. Similarly its could also be linked to the case of CDMA systems with frequency selective channels as recently shown in [15]. To conclude this remark on CDMA systems, it could be verified $[6,15]$ that the denominator of the transmit power at the NE becomes proportional to $1-\frac{\beta^{*}}{N}$, which is in favor of the existence of a non-trivial equilibrium in games under investigation.

\section{A HIERARCHICAL GAME WITH SIN- GLE USER DECODING}

As mentioned previously, one of our motivations for introducing hierarchy is to improve the network energy-efficiency. The proposed approaches can be seen as intermediate schemes between the totally centralized power control policy and the non-cooperative policy of $[5,6]$. It is also quite relevant for flexible networks where the trend is to split the intelligence between the network infrastructure and the (generally mobile) users' equipments. These approaches are therefore reasonable ways of finding a desired trade-off between the desired global network performance and amount of control signalling sent by the receiver. In this section we propose a Stackelberg formulation of the power control game where one of the (two) users is chosen to be the leader. This means that the receiver is not a player of the game. In this respect we will always assume in this section that single-user decoding is implemented at the receiver. The motivations for using SUD can be precisely that the receiver has to remain neutral in the game or/and for limiting the receiver complexity. In Sec. 6 we will however use a second Stackelberg formulation (similar to that used by [3] for water-filling games where Shannon transmission rates are considered for the users' utilities) where the receiver uses SIC and has its own utility.

Here, we consider without loss of generality (but possibly with a loss of optimality) that user 1 is the leader of the game and user 2 is the follower. The key idea is to consider that there is a cognitive user who is the follower and react after sensing the power level played by user 1 (more precisely, what needs to be known is $\left|h_{1}\right|^{2} P_{1}$ ). If such a sensing mechanism is difficult or impossible in the context of interest, another way for the follower to have this knowledge is to receive it from the base station. Indeed, if the base station broadcasts the necessary information to the users, one obtains a game which is mathematically identical (it is not equivalent physically e.g because more signalling is required from the receiver). Note that often, in the case of coherent communications, the base station has an estimate of each $\left|h_{i}\right|^{2} P_{i}$; they can be estimated, for example, by using the training sequence sent by each user. Interestingly, it is possible to show that, under realistic conditions, there is a unique equilibrium in this hierarchical game, which we call a Stackelberg Equilibrium (SE). Before indicating how the users determine their optimal transmit power let us define a Stackelberg equilibrium.

Definition 4.1 (Stackelberg EQuilibrium). A strategy profile $\left(p_{1}^{S E}, p_{2}^{S E}\right)$ is called a (pure) Stackelberg equilibrium if $p_{1}^{S E}$ maximizes the single-variable utility of the leader and $p_{2}^{S E} \in \mathrm{BR}_{2}\left(p_{1}\right)$, where the notation $\mathrm{BR}_{j}\left(p_{i}\right)$ stands for best response of player $j$ to player $i \neq j$.

By denoting $\left(p_{1}^{S E}, p_{2}^{S E}\right)$ the power profile at the SE, this definition translates mathematically as

$$
p_{1}^{S E}=\arg \max _{p_{1}} u_{1}\left(p_{1}, p_{2}\left(p_{1}\right)\right),
$$

with for all $p_{1}$,

$$
\mathrm{BR}_{2}\left(p_{1}\right)=\arg \max _{p_{2}} u_{2}\left(p_{1}, p_{2}\right)
$$

and $p_{2}^{S E}=\mathrm{BR}_{2}\left(p_{1}^{S E}\right)$. Now we look at the problem of the existence and uniqueness of such a pair of transmit power levels. A solution to these issues is stated through the following proposition.

Proposition 4.2. (Existence AND UNIQUENESS OF AN $\mathrm{SE})$. There is a unique equilibrium in the proposed hierarchical game $\left(p_{1}^{S E}, p_{2}^{S E}\right)$ :

$$
p_{1}^{S E}=\frac{\sigma^{2}}{\left|h_{1}\right|^{2}} \frac{\gamma^{*}\left(1+\beta^{*}\right)}{1-\gamma^{*} \beta^{*}} \quad \text { and } \quad p_{2}^{S E}=\frac{\sigma^{2}}{\left|h_{2}\right|^{2}} \frac{\beta^{*}\left(1+\gamma^{*}\right)}{1-\gamma^{*} \beta^{*}},
$$

if the following (sufficient) conditions hold: $\frac{f^{\prime \prime}(0)}{f^{\prime}(0)} \geq 2 \beta^{*}$ and $\phi(x)=x\left(1-\beta^{*} x\right) f^{\prime}(x)-f(x)$ has a single maximum in $] 0, \gamma^{*}\left[\right.$, where $\beta^{*}$ is the positive solution of the equation $x f^{\prime}(x)-f(x)=0$ and $\gamma^{*}$ the positive solution of the equation $\phi(x)=0$.

Proof. Using the utility function defined by Eq. (9), we obtain that for all $p_{1}$, the optimal decision for the follower, given the power of the leader, is to choose the power

$$
p_{2}^{S E}\left(p_{1}\right)=\beta^{*}\left(p_{1}\left|h_{1}\right|^{2}+\sigma^{2}\right) \frac{1}{\left|h_{2}^{2}\right|} .
$$

Plugging this value in the utility function of user 1 , we obtain:

$$
u_{1}\left(p_{1}\right)=\frac{R_{1} f\left[\frac{p_{1}\left|h_{1}\right|^{2}}{\beta^{*} p_{1}\left|h_{1}\right|^{2}+\sigma^{2}\left(1+\beta^{*}\right)}\right]}{p_{1}} \triangleq \frac{R_{1} f\left[g\left(p_{1}\right)\right]}{p_{1}} .
$$

We have that $p_{1}^{S E}$ has to verify $p_{1}^{S E} g^{\prime}\left(p_{1}^{S E}\right) f^{\prime}\left[g\left(p_{1}^{S E}\right)\right]=$ $f\left[g\left(p_{1}^{S E}\right)\right]$. This equation is equivalent to finding $p_{1}$ such that

$$
g\left(p_{1}\right) f^{\prime}\left[g\left(p_{1}\right)\right]=f\left[g\left(p_{1}\right)\right]\left(1+\frac{\beta^{*}}{1+\beta^{*}} \frac{\left|h_{1}\right|^{2}}{\sigma^{2}} p_{1}\right) .
$$

Define $x$ by $x \triangleq \frac{p_{1}\left|h_{1}\right|^{2}}{\beta^{*} p_{1}\left|h_{1}\right|^{2}+\sigma^{2}\left(1+\beta^{*}\right)}$. Studying the existence and uniqueness issues for $p_{1}$ is equivalent to analyzing those of $x_{0}$ such that $\phi\left(x_{0}\right)=0$ with

$$
\phi(x)=x\left(1-\beta^{*} x\right) f^{\prime}(x)-f(x)
$$

where:

- 1. $f$ is continuous over $[0,+\infty)$;

- 2. $f(0)=0$; 
- 3. $\forall x \geq 0, f^{\prime}(x) \geq 0$;

- 4. as $f$ is S-shaped we can define an $x_{c}$ such that $\forall x \leq x_{c}, f^{\prime \prime}(x) \geq 0$ and $\forall x \geq x_{c}, f^{\prime \prime}(x) \leq 0$;

- 5. $\lim _{x \rightarrow+\infty} f(x)=$ const.

Recall that const $=1$ in $[6]$. This property is essential to insure the existence and uniqueness of $\beta^{*}$. In fact, to be more rigorous, a condition of the corresponding convergence rate has to be added to insure $\lim _{p_{i} \rightarrow 0} u_{i}\left(p_{i}\right)=0$. Therefore our problem boils down to knowing the sign of $\phi^{\prime}(x)$ for $x \geq 0$.

Existence of $x_{0}$. We know that $\phi(0)=0$ and $\forall x \geq$ $\frac{1}{\beta^{*}}, \phi(x)<0$. Therefore if we can prove that $\mathrm{f}$ is locally strictly positive on the interval $] 0, \frac{1}{\beta^{*}}[$ the existence of $x_{0}$ will be guaranteed. A sufficient condition for the existence of $x_{0}$ is $\frac{f^{\prime \prime}(0)}{f^{\prime}(0)} \geq 2 \beta^{*}$. To check this use $\phi^{\prime \prime}(x)=$ $-2 \beta^{*} f^{\prime}(x)+f^{\prime \prime}(x)+x\left[-4 \beta^{*} f^{\prime \prime}(x)+\left(1-\beta^{*} x\right) f^{\prime \prime \prime}(x)\right]$ and call for the Taylor-Lagrange theorem: there exists $c \in] 0, x[$ such that $\phi(x)=\phi^{\prime \prime}(0) \frac{x^{2}}{2}+\phi^{\prime \prime \prime}(c) \frac{c^{3}}{6}$. The quantity $\frac{c^{3}}{x^{2}} \leq x$ can be made arbitrary small in the neighborhood of zero. The proposed sufficient condition insures the convexity of $\phi$ and $\phi$ is therefore locally strictly positive.

Uniqueness of $x_{0}$. Straightforward.

In order to have an idea to what extent the sufficient condition stated in Prop. 4.2 is realistic, we consider the practical choice of efficiency function proposed by [5] and also used by [6, 11]: $f(x)=\left(1-e^{-x}\right)^{M}$ where $M$ is the block length (this function is a reasonable model for evaluating the packet success rate of a transmission). Assuming this function, we have $\left.\phi^{\prime}(x)=e^{-x}\left[\beta^{*} x^{2}-\left(2 \beta^{*} M+1\right) x+M-1\right)\right]$ and the existence and uniqueness of $p_{1}^{S E}$ and $p_{2}^{S E}$ readily follows from the above proof.

At this point, at least two questions arise:

- From a user point of view, is it better to be chosen to be a leader or a follower?

- With respect to the Nash equilibrium what is the gain brought by introducing hierarchy? Do all the players benefit from this?

The first question is answered in Prop. 4.3 whereas the latter questions are the purpose of Prop. 4.4.

Proposition 4.3. (Following is Better than LEADING). Any user has always a better utility by being chosen as a follower than a leader.

Proof. We denote by $u_{L}^{(S E)}$ (resp. $u_{F}^{(S E)}$ ) the utility of user $i \in\{1,2\}$ when he is chosen to be the leader (resp. follower) of the game. First, we observe that we have that the SINR for the leader and the follower are: $S I N R_{L}^{(S E)}=$ $\gamma^{*}$ and $S I N R_{F}^{(S E)}=\beta^{*}$. From [14], we have that for all $x>0$

$$
x>\beta^{*} \Leftrightarrow x f^{\prime}(x)<f(x) .
$$

As for all $x>0, x\left(1-\beta^{*} x\right) f^{\prime}(x)<x f^{\prime}(x)$ from a simple geometrical argument we see that $\gamma^{*}<\beta^{*}$. This means that the SINR of the follower (i.e. $\beta^{*}$ ) is higher than the SINR of the leader (i.e. $\beta^{*}$ ). Therefore, we can write that

$$
\begin{aligned}
\frac{u_{L}^{(S E)}}{u_{F}^{(S E)}} & =f\left(\gamma^{*}\right) \frac{\left|h_{i}\right|^{2}}{\sigma^{2}} \frac{1-\beta^{*} \gamma^{*}}{\gamma^{*}\left(1+\beta^{*}\right)} \frac{1}{f\left(\beta^{*}\right)} \frac{\sigma^{2}}{\left|h_{i}\right|^{2}} \frac{\beta^{*}\left(1+\gamma^{*}\right)}{1-\beta^{*} \gamma^{*}} \\
& =\frac{\frac{f\left(\gamma^{*}\right)}{\gamma^{*}}}{\frac{f\left(\beta^{*}\right)}{\beta^{*}}} \frac{1+\gamma^{*}}{1+\beta^{*}} \\
& =\frac{h\left(\gamma^{*}\right)}{h\left(\beta^{*}\right)} \frac{1+\gamma^{*}}{1+\beta^{*}} \\
& \leq 1
\end{aligned}
$$

where the inequality follows from $\gamma^{*} \leq \beta^{*}$ and the fact that function $h: x \mapsto \frac{f(x)}{x}$ reaches its maximum in $\beta^{*}$.

The main issue we need to address now is the comparison between the non-cooperative and hierarchical games in terms of energy efficiency. Specifically we want to compare the values of $u_{i}\left(p_{1}^{S E}, p_{2}^{S E}\right)$ and $u_{i}\left(p_{1}^{N E}, p_{2}^{N E}\right)$, for each player $i \in\{1,2\}$. This is stated through the following proposition.

Proposition 4.4. (UNIFORM IMPROVEMENT OF UTILITIES). We always assume that single-user decoding is used at the receiver. Then, in the Stackelberg formulation, both the leader and follower improve their utilities with respect to the non-cooperative setting.

Proof. (i) The follower improves his utility. Denoting by $i$ the index of the follower we have:

$$
\begin{aligned}
\frac{u_{i}^{(S E)}}{u_{i}^{(N E)}} & =f\left(\beta^{*}\right) \frac{\left|h_{i}\right|^{2}}{\sigma^{2}} \frac{1-\beta^{*} \gamma^{*}}{\beta^{*}\left(1+\gamma^{*}\right)} \frac{1}{f\left(\beta^{*}\right)} \frac{\sigma^{2}}{\left|h_{i}\right|^{2}} \frac{\beta^{*}}{1-\beta^{*}} \\
& =\frac{1}{1+\frac{\gamma^{*}-\beta^{*}}{1-\beta^{*} \gamma^{*}}} \\
& \geq 1
\end{aligned}
$$

where the inequality follows from $0 \leq \gamma^{*} \leq \beta^{*}<1$.

(j) The leader improves his utility. Denoting by $j \neq i$ the index of the leader we have:

$$
\begin{aligned}
\frac{u_{j}^{(S E)}}{u_{j}^{(N E)}} & =f\left(\gamma^{*}\right) \frac{\left|h_{j}\right|^{2}}{\sigma^{2}} \frac{1-\beta^{*} \gamma^{*}}{\gamma^{*}\left(1+\beta^{*}\right)} \frac{1}{f\left(\beta^{*}\right)} \frac{\sigma^{2}}{\left|h_{j}\right|^{2}} \frac{\beta^{*}}{1-\beta^{*}} \\
& =\frac{\frac{f\left(\gamma^{*}\right)}{\gamma^{*}}\left(1-\beta^{*} \gamma^{*}\right)}{\frac{f\left(\beta^{*}\right)}{\beta^{*}}\left[1-\left(\beta^{*}\right)^{2}\right]} \\
& =\frac{g\left(\gamma^{*}\right)}{g\left(\beta^{*}\right)}
\end{aligned}
$$

where $g: x \mapsto \frac{f(x)}{x}\left(1-\beta^{*} x\right)$. It can be checked that $g^{\prime}(x)=\frac{x\left(1-\beta^{*} x\right) f^{\prime}(x)-f(x)}{x^{2}}=\frac{\phi(x)}{x}$ where $\phi$ is defined by Eq. (16). From this analysis we know that $\forall x \in\left[\gamma^{*}, \beta^{*}\right], \phi(x) \leq$ 0 , which shows that the function $g$ is non-increasing over $\left[\gamma^{*}, \beta^{*}\right]$. Therefore we have that $\frac{g\left(\gamma^{*}\right)}{g\left(\beta^{*}\right)} \geq 1$, which concludes the proof.

To conclude this section we will make two comments. First, it is very interesting to observe that both players benefit from hierarchy. This results it not usual in game-theoretic studies. In economics, for instance, in the case of a duopoly [16], only the leader can benefit from the introduction of hierarchy. As a second comment, we note that both users not only obtain a better energy-efficiency in the proposed Stackelberg game but it can also be checked that they transmit with a lower power than in the non-cooperative game (Sec. 
3 ), which could be roughly interpreted by saying that cognition incites selfish people to use their resources in a more clever manner.

\section{A HIERARCHICAL GAME WITH SUC- CESSIVE INTERFERENCE CANCELA- TION}

In this section the receiver is assumed to implement successive interference cancelation. The principle of SIC is to rank the users and decode them successively (see e.g. [1]). For the 2-user case the decoding is a two-stage process. In the first stage, the receiver decodes a user (say user 1) by considering the other (user 2) as part of the noise. In the second stage, i.e. after the first user has been decoded successfully, the first user can be subtracted from the received signal and user 2 is decoded without multiuser interference. Compared to the case of the SUD-based receiver, the SICbased does not require any additional knowledge and therefore always only use the receiver channel state information $\left(h_{1}, \ldots, h_{K}\right)$ on each block of data, just as SUD. From a practical point of view the two main differences between SIC and SUD is that SIC is more complex to be implemented and the decoding order has to be known to the users. For the latter point, as mentioned in [4], it does not necessarily mean that the receiver has to send a signal for indicating the decoding order to the user. In fact, the information can also be acquired from an external (and therefore free in terms of signalling cost) source of signal. However, there is generally in this case a loss of optimality for the overall network performance. Clearly, one of the main advantages for using a SIC at the receiver is to partially remove some multiuser interference. Note that, in the context of system with mutual user interaction, improving the decoding scheme does not always imply that each user improves his utility. It turns out it is the case in the game under investigation. We will see that using a SIC leads to a non-negative gain for the individual energy-efficiency of every user. A second nice feature of the SIC is that it unconditionally insures the existence and uniqueness of an equilibrium. All the issues we have just mentioned are precisely the purpose of this section.

Proposition 5.1. (Existence AND UniQueness of AN $\mathrm{NE}$ ). Assume $K \geq 2$. Without loss of generality assume that a user receives the index $i$ if he is decoded with rank $K-i+1$ in the successive decoding procedure at the receiver. In the non-cooperative game with a SIC-based receiver where the utility is chosen to be given by Eq. (3) where the SINRs are those considered at the output of the SIC, there exists a unique (pure) Nash equilibrium $\left(p_{1}^{(S I C)}, \ldots, p_{K}^{(S I C)}\right)$ which is given by:

$$
\forall i \in\{1, \ldots, K\}, p_{i}^{(S I C)}=\frac{\sigma^{2}}{\left|h_{i}\right|^{2}} \beta^{*}\left(1+\beta^{*}\right)^{i-1} .
$$

Proof. First note that, in the output of the SIC, the SINR of user $i$ is given by

$$
S I N R_{i}=\beta^{*}=\frac{p_{i}\left|h_{i}\right|^{2}}{\sigma^{2}+\sum_{j=0}^{i-1}\left|h_{j}\right|^{2} p_{j}} .
$$

Although we deal here with a non-linear receiver we see that every output SINR has the same key property as for linear receivers [6][11] i.e. $p_{i} \frac{\partial S I N R_{i}}{\partial p_{i}}=S I N R_{i}$. Thus, in order to maximize its utility (Eq. (3)) each user has to tune its transmit power such that its SINR is equal to $\beta^{(S I C)}=\beta^{*}$ where $\beta^{*} f^{\prime}\left(\beta^{*}\right)=f\left(\beta^{*}\right)$. Knowing this, it is easy to express the best responses of the (two) users: $\operatorname{BR}_{1}\left(p_{2}\right)=\frac{\sigma^{2}}{\left|h_{1}\right|^{2}} \beta^{*}$ and $\operatorname{BR}_{2}\left(p_{1}\right)=\frac{\left|h_{1}\right|^{2}}{\left|h_{2}\right|^{2}} \beta^{*} p_{1}+\frac{\sigma^{2}}{\left|h_{2}\right|^{2}} \beta^{*}$. In the plane of strategies $\left(p_{1}, p_{2}\right)$ these two responses have always an intersection (which insures the existence of a Nash equilibrium) and it is unique. The expressions of the transmit powers at the equilibrium follow.

As we did in Sec. 4 through Prop. 4.4 we now want to compare the energy-efficiency obtained by the users in the (SIC-based) hierarchical game with those obtained in the (SUD-based) non-cooperative game.

Proposition 5.2. (Uniform improvement of UtiliTIES). Let $K=2$. Assume $\beta^{*}<1$. Using a SIC-based receiver instead of a SUD-based receiver allows every user to improve his utility.

Proof. Without loss of generality consider user 1 . The ratio of its utility at the NE when a SUD is assumed to that obtained when a SIC is assumed is:

$$
\begin{aligned}
\frac{u_{1}^{(S I C)}}{u_{1}^{(S U D)}} & =\frac{R_{1} f\left(\beta^{*}\right)}{p_{1}^{(S I C)}} \frac{p_{1}^{(S U D)}}{R_{1} f\left(\beta^{*}\right)} \\
& =\frac{p_{1}^{(S U D)}}{p_{1}^{(S I C)}} \\
& =\frac{1}{1-\left(\beta^{*}\right)^{d}} \\
& \stackrel{(a)}{\geq} 1
\end{aligned}
$$

where $d=1$ if user 1 is decoded in second or $d=2$ if he is decoded first and inequality (a) is verified since $\beta^{*} \geq 0$. The reasoning we just made is also valid for user 2 .

At this point we have proved that in both proposed hierarchical and non-symmetric games the utility of every player is improved w.r.t. the non-cooperative game. Note that the results hold for the 2 -user case, which is the most simple case to treat and introduce the new concepts needed. Now we also want to compare the two proposed hierarchical games between themselves. More precisely, we want to compare the utilities obtained at the SE with SUD with those obtained at the NE when SIC is assumed. This comparison is obviously unfair since the reception schemes are different. However it might correspond to a question that a system designer could ask himself. The main issue is to know if there could exist scenarios where a Stackelberg game with SUD could perform better than a non-cooperative game with a superior reception scheme that mitigate interference. In other words, can a nice game theoretic formulation compensate for a suboptimal choice in terms of decoding scheme? The answer is provided in the following proposition (Prop. $5.3)$.

Proposition 5.3. (COMPARISON BETWEEN THE TWO HIERARCHICAL GAMES). For any user $i \in\{1,2\}$ we have the following results:

1. It is better for user $i$ to be decoded last in the noncooperative game with SIC than being the follower in the Stackelberg game with SUD. 
2. It is better for user $i$ to be decoded last in the noncooperative game with SIC than being the leader in the Stackelberg game with SUD.

3. It is better for user $i$ to be decoded first in the noncooperative game with SIC than being the leader in the Stackelberg game with SUD.

Proof. Notations: we always denote by $u_{L}^{(S E)}$ (resp. $u_{F}^{(S E)}$ ) the utility of user $i \in\{1,2\}$ when it is chosen to be the leader (follower) of the game. We also denote by $u_{-}^{(S I C)}$ (resp. $\left.u_{+}^{(S I C)}\right)$ the utility of user $i \in\{1,2\}$ when it is decoded in the first (resp. last) position.

(1) $\frac{u_{+}^{(S I C)}}{u_{F}^{(S E)}}=\frac{1+\gamma^{*}}{1-\beta^{*} \gamma^{*}} \geq 1$ since $0 \leq \gamma^{*} \leq \beta^{*}<1$.

(2) By Proposition 4.3, $\frac{u_{+}^{(S I C)}}{u_{L}^{(S E)}} \geq \frac{u_{+}^{(S I C)}}{u_{F}^{(S E)}} \geq 1$.

(3) $\frac{u_{-}^{(S I C)}}{u_{L}^{(S E)}}=\frac{\frac{f\left(\beta^{*}\right)}{\beta^{*}}}{\frac{f\left(\gamma^{*}\right)}{\gamma^{*}}} \frac{1}{1-\beta^{*} \gamma^{*}} \geq \frac{\frac{f\left(\beta^{*}\right)}{\beta^{*}}}{\frac{f\left(\gamma^{*}\right)}{\gamma^{*}}} \geq 1$ because $h: x \mapsto$ $\frac{f(x)}{x}$ reaches its maximum in $\beta^{*}$ and $0 \leq \gamma^{*} \leq \beta^{*}<1$.

CONJECTURE 5.4. It is better for user $i$ to be decoded first in the non-cooperative game with SIC than being the follower in the Stackelberg game with SUD if $\gamma^{*}>\frac{\beta^{*}}{1+\beta^{*}+\left(\beta^{*}\right)^{2}}$. Otherwise, user $i$ has a better utility in the Stackelberg game with SUD.

This conjecture is supported by the fact that $\frac{u_{-}^{(S I C)}}{u_{F}^{(S E)}}=\frac{1+\gamma^{*}}{1+\beta^{*}} \frac{1}{1-\beta^{*} \gamma^{*}}$ and this quantity is greater than 1 if and only if $\gamma^{*} \geq$ $\frac{\beta^{*}}{1+\beta^{*}+\left(\beta^{*}\right)^{2}}$. At the time this paper is submitted we have not proved this conjecture yet. Hopefully it will be available for the final version of this paper. The critical regime we emphasize here shows a case where a Stackelberg game for which the multiuser interference is not canceled leads for a better utility for the follower than that he would have in a system where it is canceled for the other user. But in terms of global network efficiency, the answer is not clear. We will treat such issues in the next section where we focus on measures of efficiency of the whole system and not on the individual utilities.

After analyzing the two proposed hierarchical games a legitimate question that might be asked is: Why not combining the two approaches by choosing a leader/follower in the case of a SIC-based receiver? It turns out that in SIC-based game, the Stackelberg formulation coincides with its noncooperative counterpart. Said otherwise, there is no point introducing more hierarchy in the SIC-based game. Showing this is the purpose of the following reasoning. Without loss of generality, assume that user 1 is the leader and user 2 the follower. The corresponding SE is given by Eq. (11) and (12). Now there are two possible scenarios depending on the user chosen to be decoded first/last.

- Scenario 1: The leader is decoded last. Then his SINR only depends on his power level $p_{1}$. The leader's strategy at the Stackelberg equilibrium is

$$
p_{1}^{S E}=\arg \max _{p_{1}} u_{1}\left(p_{1}\right)=\frac{\sigma^{2}}{\left|h_{1}\right|^{2}} \beta^{*}=p_{1}^{(S I C)} .
$$

This implies that, at the equilibrium, the follower's strategy (who plays after the leader) is

$p_{2}^{S E}=\arg \max _{p_{2}} u_{2}\left(p_{1}^{S E}, p_{2}\right)=\frac{\sigma^{2}}{\left|h_{2}\right|^{2}} \beta^{*}\left(1+\beta^{*}\right)=p_{2}^{(S I C)}$.

Clearly, the Stackelberg equilibrium coincides with the Nash equilibrium in this scenario.

- Scenario 2: The leader is decoded first. Then, the SINR of the follower only depends on his transmission power $p_{2}$ and his strategy at the Stackelberg equilibrium is

$$
p_{2}^{S E}=\arg \max _{p_{2}} u_{2}\left(p_{2}\right)=\frac{\sigma^{2}}{\left|h_{2}\right|^{2}} \beta^{*}=p_{1}^{(S I C)} .
$$

This corresponds to the strategy of the last decoded player in the non-cooperative game using SIC. The leader of the Stackelberg game optimizes his utility where the decision of the follower does not depend on his strategy. Actually, we observe that it is no more a Stackelberg game, as the follower decision does not depend on the leader decision. At the equilibrium, the leader's decision is:

$p_{1}^{S E}=\arg \max _{p_{1}} u_{1}\left(p_{1}, p_{2}^{S E}\right)=\frac{\sigma^{2}}{\left|h_{1}\right|^{2}} \beta^{*}\left(1+\beta^{*}\right)=p_{2}^{(S I C)}$,

which coincides with the strategy played by the user decoded first in the non-cooperative game with SIC.

\section{NETWORK ENERGY-EFFICIENCY ANAL- YSIS}

So far, in the two hierarchical games analyzed previously, we have assumed an arbitrary choice for the follower (Stackelberg game with SUD) and decoding order (non-cooperative game with SIC). In this section we want to optimize the overall network energy-efficiency with respect to the aforementioned degrees of freedom. For this purpose we consider two measures: the social welfare [17] which is well known in game theoretic studies and the energy-efficiency of the equivalent virtual multiple input multiple output (MIMO) system, the latter being used to optimize power allocation in multicarrier CDMA systems [6]. This will allow us to have two complementary points of view on the way of measuring the energy-efficiency of a network. As a comment regarding the terminology used, note that if the non-cooperative game with SIC is optimized in terms of a certain measure of energy-efficiency of the global network, the game can be seen as a Stackelberg game where: (a) the receiver is the game leader; (b) its set of strategies is the set of all decoding orders; (c) its utility is $w$ (Eq. (28)) or $v$ (Eq. (30)); (d) the users are the followers.

\subsection{Social welfare}

The social welfare of the network is measured by the total utility of the system expressed as follows:

$$
w=\sum_{i=1}^{K} u_{i}=\sum_{i=1}^{K} \frac{T_{i}}{p_{i}} .
$$

For this measure we have the following two results for the two games with 2 players. 
Proposition 6.1. (Best ChOICE OF THE FOllower). Assume a Stackelberg game with SUD. Let $K=2$. In order to maximize the social welfare the user who has the best $R_{i}\left|h_{i}\right|^{2}$ has to be chosen as the game follower.

Proof. Let $w^{(1)}$ (resp. $w^{(2)}$ ) be the social welfare when user 1 (resp. user 2) is chosen to be the follower. We have that

$$
\begin{aligned}
& w^{(1)}=R_{2} \frac{f\left(\gamma^{*}\right)\left|h_{2}\right|^{2}\left(1-\gamma^{*} \beta^{*}\right)}{\sigma^{2} \gamma^{*}\left(1+\beta^{*}\right)}+R_{1} \frac{f\left(\beta^{*}\right)\left|h_{1}\right|^{2}\left(1-\gamma^{*} \beta^{*}\right)}{\sigma^{2} \beta^{*}\left(1+\gamma^{*}\right)} \\
& w^{(2)}=R_{1} \frac{f\left(\gamma^{*}\right)\left|h_{1}\right|^{2}\left(1-\gamma^{*} \beta^{*}\right)}{\sigma^{2} \gamma^{*}\left(1+\beta^{*}\right)}+R_{2} \frac{f\left(\beta^{*}\right)\left|h_{2}\right|^{2}\left(1-\gamma^{*} \beta^{*}\right)}{\sigma^{2} \beta^{*}\left(1+\gamma^{*}\right)} .
\end{aligned}
$$

Therefore

$$
\begin{aligned}
& w^{(2)}-w^{(1)}= \\
& \frac{1-\gamma^{*} \beta^{*}}{\sigma^{2}}\left(R_{2}\left|h_{2}\right|^{2}-R_{1}\left|h_{1}\right|^{2}\right) \times\left[\frac{f\left(\beta^{*}\right)}{\beta^{*}\left(1+\gamma^{*}\right)}-\frac{f\left(\gamma^{*}\right)}{\gamma^{*}\left(1+\beta^{*}\right)}\right] .
\end{aligned}
$$

Since we know that $\frac{f\left(\beta^{*}\right)}{\beta^{*}}>\frac{f\left(\gamma^{*}\right)}{\gamma^{*}}$ and $\beta^{*}>\gamma^{*}$, we see that the difference is positive if and only if $R_{2}\left|h_{2}\right|^{2}>R_{1}\left|h_{1}\right|^{2}$, which concludes the proof.

Proposition 6.2. (Best DeCOding ORder). Assume a non-cooperative game with $S I C$. Let $K \geq 2$. The best decoding order in the sense of the social welfare is to decode the users in the increasing order of their energy weighted by the coding rate $R_{i}\left|h_{i}\right|^{2}$.

The proof of this proposition is straightforward. From these two propositions we see that using the social welfare as a global measure of efficiency always gives an advantage to the dominant user in asymmetric channels i.e. for which $\left|h_{i}\right|^{2}>>\left|h_{j}\right|^{2}$ for $i \neq j$. Indeed, in the Stackelberg game with SUD the strongest user is chosen to be the follower and in the non-cooperative game with SIC he is chosen to be decoded last. In the limit case where $\frac{\left|h_{1}\right|}{\left|h_{2}\right|} \rightarrow+\infty$, we have that

$$
\lim _{\frac{\left|h_{1}\right|}{\left|h_{2}\right|} \rightarrow+\infty} w=+\infty
$$

Therefore if one user becomes more and more satisfied the whole society becomes more and more satisfied. Clearly, the social welfare as defined by Eq. (28) is not that social one could expect in the sense it ignores unfairness. This is one of the reasons why other measures of efficiency can be used. In the next subsection we propose to consider the efficiency of the equivalent virtual MIMO system.

\subsection{Equivalent virtual MIMO network (EVMN) energy-efficiency}

We consider now another performance metric which corresponds to the energy-efficiency of an equivalent system where the transmitters would be co-located. It is described in [6] as the individual utility in the context of multi-carrier systems:

$$
v=\frac{\sum_{i=1}^{K} T_{i}}{\sum_{i=1}^{K} p_{i}} .
$$

Interestingly, one can obtain very different conclusions by optimizing this quantity with respect to the degrees of freedom instead of the social welfare. The following two propositions illustrate this point.
Proposition 6.3. (Best ChOICE OF THE FOLlOWER). Assume a Stackelberg game with SUD. Let $K=2$. Without loss of generality assume that $\left|h_{i}\right|^{2}<\left|h_{j}\right|^{2}, i \neq j$. In order to maximize energy efficiency of the equivalent virtual MIMO user $i$ (resp. $j$ ) has to be chosen as the game leader if $a R_{i} \leq R_{j}$ (resp. $a R_{i} \geq R_{j}$ ) where $a<1$ is defined by $a \triangleq$ $\frac{f\left(\beta^{*}\right) \alpha_{2}-f\left(\gamma^{*}\right) \alpha_{1}}{f\left(\beta^{*}\right) \alpha_{1}-f\left(\gamma^{*}\right) \alpha_{2}}$ with $\alpha_{1}=\left|h_{i}\right|^{2} \gamma^{*}\left(1+\beta^{*}\right)+\left|h_{j}\right|^{2} \beta^{*}\left(1+\gamma^{*}\right)$ and $\alpha_{2}=\left|h_{j}\right|^{2} \gamma^{*}\left(1+\beta^{*}\right)+\left|h_{i}\right|^{2} \beta^{*}\left(1+\gamma^{*}\right)$.

Proof. Note that $a<1$ since $\beta^{*}>\gamma^{*}$ and $\left|h_{i}\right|^{2} \leq\left|h_{j}\right|^{2}$. Let $v^{(k)}$ be the EVMN when user $k$ is the leader. From the definition of $v$ we have

$$
\begin{aligned}
v^{(i)} & =\frac{R_{i} f\left(\gamma^{*}\right)+R_{j} f\left(\beta^{*}\right)}{p_{t o t}^{(i)}} \\
v^{(j)} & =\frac{R_{j} f\left(\gamma^{*}\right)+R_{i} f\left(\beta^{*}\right)}{p_{t o t}^{(j)}}
\end{aligned}
$$

where $p_{\text {tot }}^{(k)}$ is the total power at equilibrium when user $k$ is the game leader. Thus

$$
\begin{aligned}
& v^{(i)}-v^{(j)}=\frac{R_{i} f\left(\gamma^{*}\right)+R_{j} f\left(\beta^{*}\right)}{p_{\text {tot }}^{(i)}}-\frac{R_{i} f\left(\beta^{*}\right)+R_{j} f\left(\gamma^{*}\right)}{p_{\text {tot }}^{(j)}} \\
= & \frac{R_{i}\left[f\left(\gamma^{*}\right) p_{\text {tot }}^{(j)}-f\left(\beta^{*}\right) p_{\text {tot }}^{(i)}\right]+R_{j}\left[f\left(\beta^{*}\right) p_{\text {tot }}^{(j)}-f\left(\gamma^{*}\right) p_{\text {tot }}^{(i)}\right]}{p_{\text {tot }}^{(i)} p_{\text {tot }}^{(j)}}
\end{aligned}
$$

Hence, this term is positive if user $i$ is chosen as the leader. This occurs when

$$
R_{j}\left[f\left(\beta^{*}\right) p_{\text {tot }}^{(j)}-f\left(\gamma^{*}\right) p_{\text {tot }}^{(i)}\right] \geq R_{i}\left[f\left(\gamma^{*}\right) p_{\text {tot }}^{(j)}+f\left(\beta^{*}\right) p_{\text {tot }}^{(i)}\right]
$$

As we have $p_{\text {tot }}^{(i)}=\frac{\sigma^{2}}{1-\gamma^{*} \beta^{*}} \frac{\alpha_{2}}{\left|h_{i} h_{j}\right|^{2}}$ and $p_{\text {tot }}^{(j)}=\frac{\sigma^{2}}{1-\gamma^{*} \beta^{*}} \frac{\alpha_{1}}{\left|h_{i} h_{j}\right|^{2}}$ the condition above (Eq. (33)) is equivalent to

$$
R_{i}\left[\frac{f\left(\beta^{*}\right) \alpha_{2}-f\left(\gamma^{*}\right) \alpha_{1}}{f\left(\beta^{*}\right) \alpha_{1}-f\left(\gamma^{*}\right) \alpha_{2}}\right] \leq R_{j},
$$

which concludes the proof.

Proposition 6.4. (BeSt DECOding ORder). Assume a non-cooperative game with SIC. Let $K \geq 2$. The best decoding order in the sense of the equivalent virtual MIMO network energy-efficiency is to decode the users in the decreasing order of their signal-to-noise ratio (SNR) $\frac{\left|h_{i}\right|^{2}}{\sigma^{2}}$.

Proof. Let $\pi \in \mathcal{P}$ be a permutation operator corresponding to the choice of the decoding order. Since the users have the same SINR at the equilibrium we have that $w=\frac{f\left(\beta^{*}\right) \times \sum_{i=1}^{K} R_{i}}{\sum_{i=1}^{K} p_{i}^{S T C}}$. Therefore,

$$
\pi^{*}=\arg \max _{\pi \in \mathcal{P}} w^{(\pi)}=\arg \min _{\pi \in \mathcal{P}} \sum_{i=1}^{K} p_{i}^{(S I C)} .
$$

As $p_{i}^{(S I C)}=\frac{\sigma^{2}}{\left|h_{i}\right|^{2}} \beta^{*}\left(1+\beta^{*}\right)^{i-1}$ it is clear that the influence of a user on the sum of powers decreases with its decoding rank. To minimize the total system power one has to decode the users with a decreasing order of their SNR.

In order to establish a connection between the social efficiency measure used in game theory $(w)$ and that used as a bound of a cooperative communication system $(v)$ we now look at the properties of these measures of network energyefficiency. Note that the main difference we have between 
our situation and that of multi-carrier systems [6] is that it is not possible here to optimally allocate the total power between the different users i.e. the power is not a transferable utility from a user to another one.

1. Uniform improvement of utilities. If the utilities of users 1 and 2 are improved as follows $u_{1}$ to $u_{1}^{\prime} \geq u_{1}$ and $u_{2}$ to $u_{2}^{\prime} \geq u_{2}$ then the social welfare is also improved as $w$ to $w^{\prime} \geq w$. Assuming the optimal choice of the follower/leader or first/last decoded user in the corresponding game, a sufficient condition for the EVMN energy-efficiency $v$ for having this property is $\gamma^{*} \geq \frac{\beta^{*}}{1+\beta^{*}+\left(\beta^{*}\right)^{2}}$.

2. Behavior for asymmetric channels. We already know that $\lim _{\left|h_{1}\right| \rightarrow+\infty} w=+\infty$. An equivalent of $v$ when $\frac{\left|h_{1}\right|}{\left|h_{2}\right|} \rightarrow+\infty$

$\frac{\left|h_{1}\right|}{\left|h_{2}\right|} \rightarrow+\infty$ is $v \sim \frac{\left|h_{2}\right|^{2}}{\sigma^{2}} \beta^{*}\left(R_{1}+R_{2}\right) f\left(\beta^{*}\right)$. Now, with the latter measure of energy-efficiency, if a user increases his pay-off, the MIMO network does not improve.

3. Resource sharing. The EVMN energy-efficiency is a fairer performance criterion than the social efficiency for the SIC but not for the Stackelberg with SUD.

- Case of SIC. Maximizing $w$ over the choice of decoding order amounts to giving more to the richest user in terms of $R_{i}\left|h_{i}\right|^{2}$. Maximizing $v$ over the choice of decoding order amounts to giving more to the poorest user in terms of $\left|h_{i}\right|^{2}$.

- Case of Stackelberg with SUD. Maximizing $w$ over the follower/leader choice amounts to giving more to the richest user in terms of $R_{i}\left|h_{i}\right|^{2}$. Maximizing $v$ over the follower/leader choice amounts to giving more to the richest user in terms of $\left|h_{i}\right|^{2}$.

\section{NUMERICAL ILLUSTRATIONS}

The main objective of this section is to evaluate numerically the relative gain brought by the introduction of hierarchy with respect to the non-cooperative game. To this end we introduce the quantity

$$
U_{i}=\frac{u_{i}-u_{i}^{N E}}{u_{i}^{N E}}
$$

where $u_{i}=u_{i}^{S E}$ or $u_{i}=u_{i}^{S I C}$, depending on the hierarchical game under consideration. Similarly, we define $W$ and $V$ to measure the relative gain in terms of global performance. We consider the efficiency function proposed in most papers dealing with power allocation games that is:

$$
f(x)=\left(1-e^{-x}\right)^{M}
$$

where $M=100$ is the block length in bits. We assume a CDMA system with a spreading factor $N \in\{8,16,32\}$. The thermal noise power $\sigma^{2}$ is chosen to be equal to $5 \times 10^{-16} \mathrm{~W}$. As mentioned at the end of Sec. 3, in CDMA systems using the mentioned spreading codes the denominator of the transmit power at the NE becomes proportional to $1-\frac{\beta^{*}}{N}$ and therefore what matters for the existence of the $\mathrm{NE}$ is that $\frac{\beta^{*}}{N}<1$. For the setup presented here $\beta^{*}=6.48$ and therefore we have that the latter condition is met for $N \in\{8,16,32\}$.

\subsection{Uniform improvement of utilities}

Assume $K=2$. We consider that the transmission rates for the first and second users are respectively $R_{1}=100 \mathrm{kbps}$ and $R_{2}=140 \mathrm{kbps}$. Assuming the Stackelberg formulation with SUD, Fig. 1 represents the relative gain in [\%] in terms of individual utility as a function of the spreading factor for each user. Clearly both users can benefit from a significant performance gain, especially for small values of the load $\frac{K}{N}$. If SIC is assumed at the receiver, the gains of the hierarchical approach over the non-cooperative approach are even more significant as shows Fig. 2.

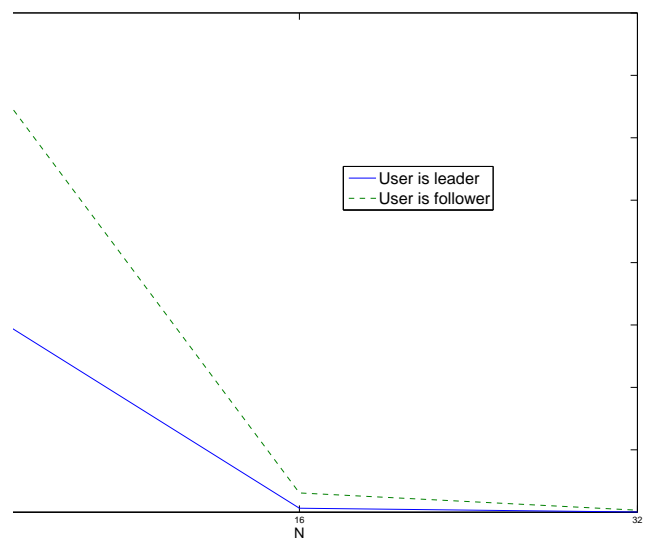

Figure 1: Individual relative performance gains (w.r.t. the non-cooperative game) for the 2 users when a Stackelberg with SUD is assumed at the receiver.

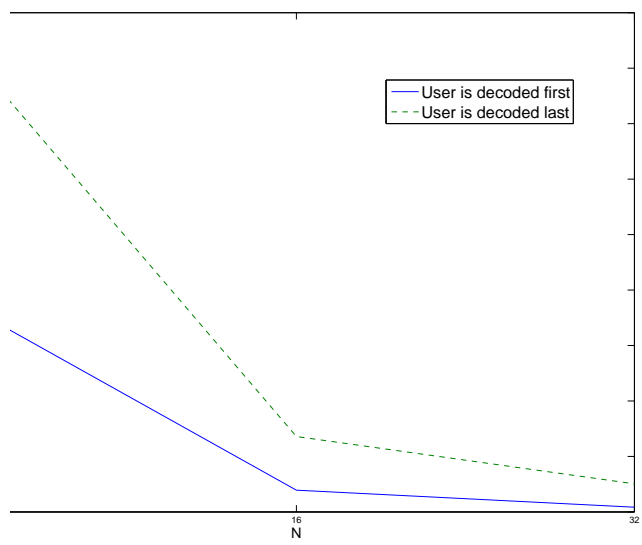

Figure 2: Individual relative performance gains (w.r.t. the non-cooperative game) for the 2 users when SIC is assumed at the receiver.

\subsection{Comparison between the two hierarchical games}

We now evaluate the relative performance gain brought by our approaches in terms of social welfare and EVMN 
energy-efficiency. The channel gains are fixed and chosen to be $\left|h_{1}\right|=2.23 \times 10^{-6}$ and $\left|h_{2}\right|=c\left|h_{1}\right|$ where $c$ represents the asymmetry of the 2 -user MAC. First, we consider the hierarchy game based on the Stackelberg formulation with SUD a the receiver and plot the social welfare and EVMN efficiency as a function of $c$. As the channel becomes more and more asymmetric the choice of the leader becomes more and more influential, in particular for extreme values of $c$. For example, in Fig. 3(c), the worse choice of the leader induces a negative relative gain for the EVMN efficiency (when $c>2$ or $c<0.8$ ). When the spreading factor increases, the benefit from choosing the best leader is a more crucial choice for the EVMN efficiency than the social welfare. We have also plotted the social welfare and EVMN energy-efficiency in the case of the hierarchical game with SIC as a function of $c$ on Fig. 4. We observe in the SIC-based game the same behavior as in the Stackelberg game with SUD. For both hierarchical games, the relative gain in terms of global efficiency obtained by introducing the hierarchy with the best choice of leader or decoding order can bring very significant performance gains if the MAC is asymmetric. We also observe that the most significant improvements are obtained for small values of the system load $\frac{K}{N}$.

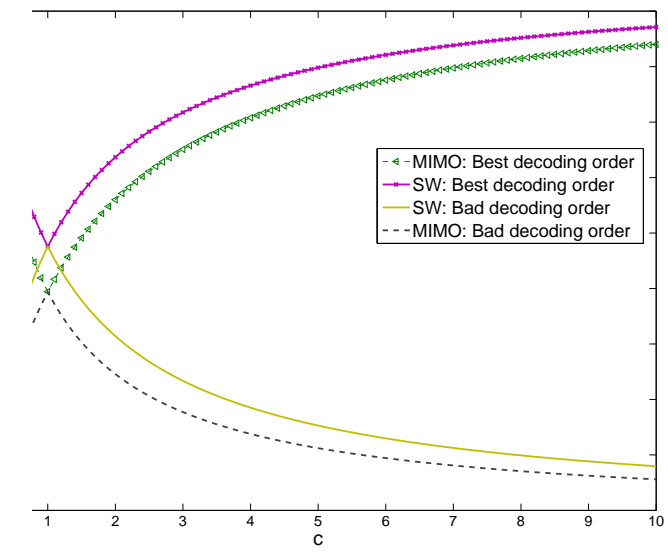

Figure 4: Global relative performance gains (w.r.t. the non-cooperative game) for the 2 users when SIC is assumed at the receiver with $N=16$.

\section{CONCLUSION}

In this work, we have analyzed the effect of hierarchy in energy-efficient power control games both on the individual performance of a given user and the overall network performance. Interestingly, the existence and uniqueness of equilibria in the considered games is insured under reasonable assumptions. In fact, when assuming successive multiuser interference cancelation at the receiver, which reduces the interaction between some users, the existence and uniqueness of a Nash equilibrium is always guaranteed. We have shown that is is also possible, at least for the 2-player game, to characterize completely and analytically the efficiency of these equilibria. Compared to most existing analyses conducted in other fields (e.g. in economics) for which game theory is applied, some unusual results have been obtained. In particular it is shown that both the leader and follower benefit from hierarchy and that social welfare can be an unfair measure of energy-efficiency of the global network. We believe that the approaches that consist in devising networks where intelligence is split in a clever manner between the network infrastructure and (generally mobile) terminals is a good way of achieving good performance in terms of energy-efficiency while achieving a desired target in terms of signalling cost. In this respect, the case of the SIC receiver clearly shows that the coordination signal does not need necessarily to be sent by the receiver but can be generated from a source external to the game (for instance from an FM signal). Relevant extensions of the presented work would consist in: (a) Extending the proposed approach to the case of networks with an arbitrary number of users. For the Stackelberg game with SUD in particular, the problem can be formulated in several ways, depending whether only one leader is designated or a class of leaders is assumed; (b) Deriving tight bounds on the price of anarchy of the network with possibly different measures of efficiency; (c) Assuming multi-carrier systems; (d) Studying the case of several receivers and the way they have to interact (e.g. the hierarchical uplink multi-cell game); (e) Analyzing the impact of channel uncertainty on the users' behavior and individual performance.

\section{REFERENCES}

[1] T. Cover and J. Thomas, "Elements of Information Theory", Wiley Interscience, 2nd edition, 2006.

[2] W. Yu, G. Ginis and J. M. Cioffi, "Distributed multiuser power control for digital subscriber lines", IEEE Journal of Selected Areas in Communications, Vol. 20, No. 5, June 2002, pp. 1105-1115.

[3] L. Lai and H. El Gamal, "The Water-Filling Game in Fading Multiple-Access Channels", IEEE Trans. on Information Theory, Vol. 54, No. 5, May 2008, pp. 2110-2122.

[4] E. V. Belmega, S. Lasaulce and M. Debbah, "Power Control in Distributed Multiple Access Channels with Coordination", IEEE Proc. of the Intl. Workshop on Wireless Networks: Communication, Cooperation and Competition (WNC3), Berlin, Germany, April 2008, pp. 1-8.

[5] D. J. Goodman and N. B. Mandayam, "Power Control for Wireless Data", IEEE Personal Communications, Vol. 7, No. 2, April 2000, pp. 48-54,

[6] F. Meshkati, M. Chiang, H. V. Poor and S. C. Schwartz, "A game-theoretic approach to energy-efficient power control in multi-carrier CDMA systems", IEEE Journal on Selected Areas in Communications, Vol. 24, No. 6, June 2006, pp. 1115-1129.

[7] C. U. Saraydar, N. B. Mandayam and D. J. Goodman, "Efficient power control via pricing in wireless data networks", IEEE Trans. on Communications, Vol. 50, No. 2, Feb. 2002, pp .291-303.

[8] B. A. Fette, "Cognitive Radio Technology", Newnes editors, 2006.

[9] I. Akyildiz, W. Lee, M. Vuran and S. Mohanty, "Next Generation/Dynamic Spectrum Access/Cognitive Radio Wireless Networks: A Survey", Computer Networks, Vol. 50, No. 13, 2006.

[10] M. Bloem, T. Alpcan and T. Basar, "A Stackelberg 


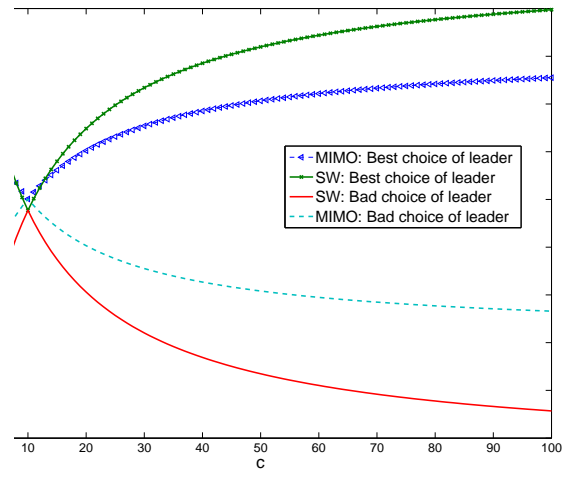

(a) $N=8$

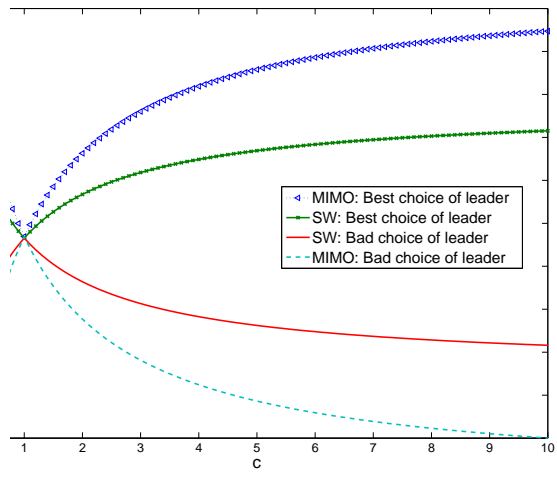

(b) $N=16$

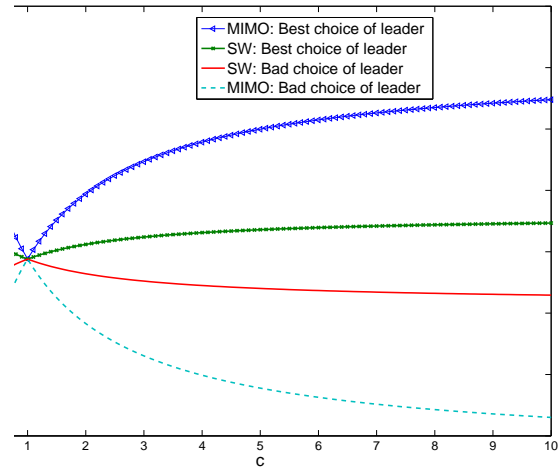

(c) $N=32$

Figure 3: Global relative performance gains (w.r.t. the non-cooperative game) for the 2 users when a Stackelberg formulation with SUD is assumed.

Game for Power Control and Channel Allocation in Cognitive Radio Networks", ACM/ICST Proc. of Gamecomm, 2007.

[11] F. Meshkati, H. Poor and S. Schwartz, "Energy-Efficient Resource Allocation in Wireless Networks", IEEE Signal Processing Magazine, Vol. 58, May 2007, pp. 58-68.

[12] T. Basar and R. Srikant, "A Stackelberg network game with a large number of followers", Journal of Optimization Theory and Applications, Vol. 115, No. 3, 2002.

[13] Y. Hayel, D. Ros and B. Tuffin, "Less-than-Best-Effort Services: Pricing and Scheduling", IEEE Proc. of INFOCOM, 2004.

[14] V. Rodriguez, "An Analytical Foundation for Resource Management in Wireless Communication", in the IEEE Proc. of Globecom, 2003.

[15] N. Bonneau, M. Debbah, E. Altman and A. Hjørungnes, "Non-Atomic Games for Multi-User System", IEEE Journal on Selected Areas in Communications: Special Issue on Game Theory in
Communication Systems, Dec. 2008, to appear.

[16] J. Hamilton and S. Slutzky, "Endogenous timing in duopoly games: Stackelberg or Cournot equilibria", Games and Economic Behavior, Vol. 2, 1990, pp. 29-46.

[17] K. J. Arrow "Social choice and individual values", Yales University Press, 1963. 